\title{
Agroforestación: una alternativa a la forestación de tierras agrarias para la domesticación del paisaje rural
}

\author{
Agroforestation: an alternative to afforestation of agricultural land \\ for landscape domestication
}

\author{
Bertomeu, M. ${ }^{1 *}$; Torres, M.'; Pulido, F.'; Moreno, G. '; Giménez, J. C. ${ }^{1}$ \\ ${ }^{1}$ INDEHESA, Escuela de Ingeniería Forestal y del Medio Natural, Centro Universitario de Plasencia, \\ Universidad de Extremadura. Avda. Virgen del Puerto 2, 10600 Plasencia, Cáceres \\ ${ }^{2}$ Miter Arbórea S.A. C/ Comandante Fortea 13, Madrid.
}




\title{
Resumen
}

Tras más de cinco décadas en las que la superficie forestal arbolada de España no ha dejado de aumentar, el reto hoy es la gestión sostenible de estos recursos forestales. Un nuevo programa de forestación de terrenos agrícolas no debería por tanto preocuparse, como en los programas precedentes, de aspectos técnicos o de aumentar la superficie forestal. Las nuevas iniciativas que busquen restaurar y diversificar el paisaje rural deberían plantear las preguntas fundamentales de quién, dónde, para qué y cómo se va a establecer el nuevo arbolado en el paisaje rural. En este artículo proponemos la agroforestación, definida como el establecimiento por agricultores de árboles y/o arbustos en terrenos agrícolas como una estrategia más eficaz que la forestación para mejorar los servicios ambientales de los agroecosistemas. La agroforestación debe basarse en 4 conceptos fundamentales: 1) La comarca geográfica, considerada como un territorio con elementos biofísicos y naturales, socio-económicos y políticos característicos e interrelacionados; 2) La domesticación del paisaje, entendida como el proceso de selección de ciertos atributos ecosistémicos deseables y la consecuente alteración de otros considerados menos deseables; 3) La agroforestería, como ciencia interdisciplinar que trata de las prácticas de uso del suelo en las que se integran especies leñosas con cultivos y/o ganado, de sus interacciones y de los beneficios que proporcionan, y 4) La participación, o proceso por el que los agricultores y propietarios, en vez de ser considerados simples beneficiarios, forman parte activa en el diseño de estrategias, en la toma de decisiones y en la gestión.

Palabras clave: agroecosistema, agroforestería, repoblación forestal, servicios ecosistémicos, participación.

\begin{abstract}
After more than five decades in which forest cover in Spain has been steadily growing because of afforestation, the challenge today is the sustainable management of the new forest resources. Therefore the main focus of a new afforestation initiative should not be further expansion of afforested land nor addressing technical issues as in previous programs. Instead a new initiative to restore and diversify rural landscapes should respond the key questions (i.e., the who, the where, the what for and the how) of afforestation. In this paper agroforestation, defined as a land rehabilitation approach through farmer-led tree planting, is proposed as a more effective strategy than afforestation to improve ecosystems services in agroecosystems. An agroforestation approach should be based on four key concepts: 1) The landscape, defined as a territory that results from the interactions between its biophysical elements with the people and their institutions and values; 2) Landscape domestication, understood as the selection of certain desirable ecosystem attributes and the consequent alteration of other ecosystem attributes that are less desirable; 3 ) Agroforestry, as an interdisciplinary science that study land-use systems where woody perennials, agricultural crops and/or animals are deliberately used on the same land simultaneously or in temporal sequence; 4) Participation, or the process by which farmers and other stakeholders are involved in program planning and management.
\end{abstract}

Keywords: agroecosystem, agroforestry, forestation, ecosystem services, participation 


\section{Introducción}

Durante el siglo XX la repoblación forestal ha sido el remedio estándar a los problemas económicos y ambientales causados por la secular deforestación. La escasez de madera, de leñas y de otras materias primas, la erosión, la degradación de suelos y la desertización, así como las avenidas e inundaciones, han sido paliadas mediante extensas repoblaciones y restauraciones hidrológico-forestales. Gracias a esta ingente actividad repobladora, así como a la recolonización por la vegetación forestal de terrenos agrícolas abandonados, desde finales del siglo XIX se produjo en gran parte de Europa y en Norteamérica, y más recientemente en China, Vietnam y en partes de la India, un aumento de la superficie forestal arbolada o una transición forestal (Mather and Needle, 1998; Mather, 2004; Mather, 2007). Globalmente, la repoblación anual de 6,8 millones de hectáreas entre 1990 y el año 2000 , y de 7,3 millones de hectáreas entre 2000 y 2005, compensó en gran medida la deforestación ocurrida en esos mismos periodos de 9,5 y 13,5 millones de hectáreas anuales respectivamente (FAO and JRC, 2012).

La actividad repobladora en España ha sido, en cuanto a superficie plantada, de las más importantes de Europa. Desde 1879 hasta hoy la superficie repoblada ha superado los 5 millones de hectáreas, lo que supone el 10\% del territorio nacional o el 18\% de la superficie forestal (Pemán y Vadell, 2009). Una parte importante de este esfuerzo repoblador corresponde a los recientes programas de Forestación de Tierras Agrícolas (FTA) llevados a cabo entre 1994 y 2009, que han supuesto la forestación de 685.000 ha con especies forestales de coníferas y frondosas en los paisajes agrícolas del centro y sur peninsular principalmente (MAPA, 2006). Así, entre 1990 y 2015, la superficie forestal en España creció a un ritmo de 184.000 ha anuales, lo que supuso un incremento de la misma de un 1,2\%, la mayor tasa de crecimiento de Europa (Forest Europe, 2015).

Actualmente existe un renovado interés en la repoblación forestal de terrenos y paisajes agrícolas debido a la urgente necesidad de mitigar los graves problemas ambientales a los que nos enfrentamos (el cambio climático, la pérdida de biodiversidad, la degradación del suelo, la contaminación atmosférica y del agua). Se calcula que en el mundo hay más de 2000 millones de hectáreas degradadas susceptibles de ser repobladas, de las que 500 millones corresponden a bosques degradados y 1500 millones de hectáreas a paisajes en mosaico con distintos grados de cobertura arbórea (Laestadius et al., 2012). Los árboles son elementos clave para el funcionamiento de los agroecosistemas que generan servicios ecosistémicos a múltiples escalas (Manning et al., 2006). Su integración en parcelas y explotaciones agrícolas puede mejorar la productividad directamente, e indirectamente mediante el control de la erosión y mejora de la fertilidad del suelo, la creación de condiciones microclimáticas favorables a los cultivos (reducción de la velocidad del viento, regulación de temperaturas extremas), y como refugio de especies beneficiosas para el control de plagas. El arbolado contribuye además a crear paisajes más diversos y atractivos que los paisajes eminentemente agrícolas o las plantaciones forestales monoespecíficas, favoreciendo la biodiversidad, sirviendo como corre- 
dores ecológicos y generando oportunidades para el turismo (Shibu, 2009; Pereira and Rodríguez, 2010; Barral et al., 2015; Torralba et al., 2016).

En España, a pesar del notable aumento de la superficie forestal arbolada de las últimas décadas, existe todavía una gran superficie de terrenos y paisajes agrícolas con escasa o nula presencia de arbolado, y por tanto, con potencial para la forestación. En un estudio para la clasificación de los paisajes de las distintas regiones bioclimáticas de España según los tipos de uso y cubiertas, un 28\% de los 412 paisajes representativos estudiados (114 paisajes) fueron clasificados como "paisajes agrícolas", mientras que sólo el 11\% (46 paisajes) fueron "paisajes agroforestales" (García del Barrio et al., 2003). Según el INE (2016), existen en nuestro país 30 millones de ha de tierras agrícolas, de las que 23 millones son tierras labradas y pastos permanentes o «superficie agrícola utilizada» (SAU), mientras que el resto, casi 7 millones de hectáreas, son "otras tierras" que formando parte de la explotación no son SAU. La introducción de árboles en estos paisajes agrícolas y agroforestales podría mejorar la producción de los cultivos y la resiliencia de los agroecosistemas, y facilitar la transición hacia una agricultura más sostenible y multifuncional.

La gran superficie de terrenos agrícolas forestados en los últimos 20 años en España induce a pensar que la FTA es una estrategia adecuada para una nueva iniciativa restauradora con el fin de diversificar los paisajes agrícolas. Sin embargo, existen importantes razones para pensar que esto no es así. En primer lugar, el éxito de la FTA no puede valorarse según simples criterios técnicos, como el número de hectáreas plantadas, o de eficiencia, como el coste por hectárea. En su lugar deberían realizarse estudios completos del impacto ambiental y socioeconómico del programa considerando un amplio rango de indicadores ecológicos, económicos y sociales (Wortley et al., 2013). Por ejemplo, en comarcas agrícolas en las que se pretende aumentar la recarga de acuíferos mediante la forestación a densidades moderadas (Ilstedt et al., 2016), existe el riesgo de que los beneficiarios utilicen las primas compensatorias recibidas por la forestación de sus parcelas menos productivas (eriales, baldíos) para intensificar la producción agrícola en otras parcelas mediante la extracción de agua para el riego. En este caso, las primas recibidas por la forestación se convertirían en un incentivo perverso (McNeely, 1998), y una valoración del éxito de la forestación basada simplemente en indicadores como la superficie reforestada, el porcentaje de marras o el número de beneficiarios, estaría muy lejos de estimar el impacto ambiental y socio-económico real de la forestación.

Conocer el impacto de la FTA en el balance de los diferentes servicios ecosistémicos supone, además, una necesaria ayuda y guía para su futura gestión. La falta de estudios amplios de los programas de FTA en España pone, cuanto menos, en cuestión su "éxito".

En segundo lugar, los programas de FTA no respondían, o lo hacían sólo parcialmente, las preguntas fundamentales que un plan de restauración de la vegetación debería plantearse:

a) ¿Por qué y para qué la forestación? 
b) ¿Dónde realizarla?

c) ¿Cómo?

d) ¿Por quién y para quién?

En consecuencia, los programas de FTA (Montiel y Galiana, 2004):

a) Carecían de un objetivo claro y formulado en respuesta a un problema concreto, lo que dio lugar a la realización de plantaciones en zonas con alto valor ecológico como atochares, o en parcelas sin las condiciones adecuadas para el establecimiento del arbolado.

b) Carecían de una escala espacial de planificación claramente definida, con lo que las forestaciones se llevaron a cabo como "un conjunto desordenado de actuaciones puntuales y desarticuladas".

c) Ofrecían un diseño estándar de plantación forestal y de su gestión que no obedecía ni a las necesidades, ni al interés de los propietarios, lo que probablemente causó un desinterés en el mantenimiento de las plantaciones y su abandono.

d) No establecieron a priori un criterio claro de selección de participantes, con lo que beneficiaron igualmente a agricultores de diverso tipo (en activo y retirados), como a grandes propietarios urbanos y a la élite rural, así como a intermediarios entre la Administración y los propietarios.

Por último, los programas sectoriales de forestación son claramente insuficientes para abordar los actuales problemas ambientales de naturaleza "perversa". El concepto de "problema perverso" surgió hace más de 40 años para diferenciar los problemas 'fácilmente' definibles, delimitables, y con solución posible, del ámbito de las ciencias naturales y de la ingeniería, de los complejos problemas sociales, como la pobreza o el desempleo, que son abordados desde la planificación y políticas públicas (Rittel and Webber, 1973; deFries and Nagendra, 2017). Debido a la gran complejidad que los caracteriza, los problemas perversos no pueden formularse de forma definitiva y no tienen una solución única, ni óptima, ni definitiva. La mayoría de los problemas relacionados con los recursos naturales, incluidos los generados por la agricultura, son 'problemas perversos' (Balint et al., 2011). Abordarlos requiere de intervenciones integradas de carácter multidisciplinar y de diversa naturaleza (técnica, económica, social, ambiental), que incluyan procesos de participación y de negociación, de generación y difusión del conocimiento, de formación y educación, y de legislación (Reed et al., 2016). Parece por tanto claro que para compatibilizar la agricultura con la generación de servicios ambientales, las estrategias sectoriales, como los programas de FTA, son claramente insuficientes.

En este trabajo proponemos, la agroforestación, definida como el establecimiento por agricultores y ganaderos de árboles y/o arbustos en terrenos y explotaciones agrícolas, como una estrategia más eficiente y sostenible que la forestación convencional para conseguir agroecosistemas multifuncionales (Roshetko et al., 2007) (Tab. 1). El concepto de agroforestación fue utilizado por primera vez en el 
Tabla 1. Diferencias más importantes entre el programa de Forestación de tierras agrícolas y la estrategia de Agroforestación.

\begin{tabular}{|c|c|c|}
\hline Característica & Forestación & Agroforestación \\
\hline Escala espacial & Parcela & Comarca geográfica \\
\hline Toma de decisiones & Autoridades Forestales & Participativo \\
\hline Estrategia & Sectorial (forestal) & $\begin{array}{c}\text { Agroforestal } \\
\text { ("El árbol apropiado en el lugar adecuado") }\end{array}$ \\
\hline Objetivos & Cambio en el uso del suelo & Multifuncionalidad \\
\hline Meta (a largo plazo) & Masas forestales & Domesticación del paisaje agrario \\
\hline Actuación prioritaria & $\begin{array}{c}\text { Plantación con especies } \\
\text { "forestales" }\end{array}$ & $\begin{array}{l}\text { Variedad de opciones y de especies: } \\
\text { setos; bosquetes o islotes; plantaciones lineales; } \\
\text { restauración de riberas; árboles aislados y } \\
\text { dispersos; prácticas de conservación del suelo } \\
\text { y del agua; prácticas agroforestales }\end{array}$ \\
\hline Enfoque disciplinar & Forestal & Multidisciplinar \\
\hline Marco institucional & Sectorial & Multisectorial \\
\hline
\end{tabular}

Sureste Asiático para denotar la rehabilitación por pequeños agricultores de extensas zonas deforestadas mediante el establecimiento de sistemas agroforestales. A pesar de las grandes diferencias ambientales, socio-económicas y culturales, el hecho de que en Europa (EU 27) y en España existan, respectivamente, 15,4 millones de hectáreas (el $8,8 \%$ de la SAU) y 5,5 millones de ha (el $23,5 \%$ de la SAU) de sistemas agroforestales (den Herder et al., 2017) nos hace pensar que la agroforestación es un concepto igualmente aplicable en nuestro contexto.

En los siguientes apartados presentamos los cuatro conceptos fundamentales en los que se sustenta la estrategia de agroforestación. Primero proponemos la "comarca geográfica" como la escala espacial de planificación de la agroforestación. Después proponemos el concepto de "domesticación del paisaje" como la meta a largo plazo de la agroforestación. En tercer lugar presentamos los sistemas y prácticas agroforestales como una forma más apropiada que las plantaciones forestales para restaurar el arbolado en terrenos agrícolas. Finalmente, introducimos el concepto de la "participación" como condición necesaria para el éxito y la sostenibilidad de la agroforestación en los paisajes agrícolas.

\section{La comarca geográfica como escala espacial para la planificación}

En España, la comarca forestal ha sido tradicionalmente la escala espacial de planificación de la repoblación forestal y de los programas de FTA (Serrada et al., 2005). Las comarcas forestales se definen como "terrenos forestales continuos con una marcada unidad topográfica" (altas cuencas hidrográficas, fondos de valle, páramos, etc.) (Reque y Pérez, 2011). Estas se identifican y delimitan mediante estudios de carácter litológico, fisiográfico y de vegetación (Serrada et al., 2005). 
Mientras que el concepto de comarca forestal ha podido ser útil para la planificación de las extensas repoblaciones forestales llevadas a cabo en los montes, sierras y cabeceras de cuencas durante casi toda la segunda mitad del siglo XX, creemos que lo es menos para la planificación de la restauración de terrenos agrícolas. Esto es debido a que su definición y estudio se centra exclusivamente en el medio físico, sin considerar que los bosques y los agroecosistemas son sistemas socio-ecológicos, y que por ello hay que considerar a la población en todos sus aspectos. Por tanto creemos que para las actividades de restauración en terrenos agrícolas es necesario definir una escala de planificación más completa.

Aspirar a la multifuncionalidad de los agroecosistemas requiere de un espacio lo suficientemente amplio para la planificación de las diversas actividades de agroforestación que satisfagan las distintas necesidades y funciones, y en el que llevar a cabo las negociaciones y compromisos que inevitablemente conllevan los diversos usos del suelo de ese espacio. En este sentido proponemos la comarca geográfica, definida como un territorio de ámbito supramunicipal que amalgama elementos naturales bióticos y abióticos (relieve, los ríos, el suelo, el clima, plantas y animales) con elementos sociales, políticos y culturales (poblaciones, instituciones, infraestructuras) característicos y con sentido de identidad y pertenencia (Mata et al., 2004), como una escala de análisis y gestión eficaz para abordar la multifuncionalidad de los agroecosistemas. La comarca geográfica presenta normalmente variaciones de altitud, roca madre y tipos de suelo, distintos hábitats o tipos de vegetación, y múltiples corrientes de agua o ríos, y poblaciones, y diferentes actividades económicas conectadas a través de los mercados locales, redes sociales, instituciones y sistemas de gobernanza. En definitiva, las comarcas son sistemas socio-ecológicos con base territorial que resultan de las interacciones entre la población y otros elementos bióticos, los factores abióticos, las instituciones (con sus leyes y normativa) y los valores de cada individuo. La comarca proporciona una escala que capta la compleja matriz de explotaciones agrícolas, familias, comunidades y ecosistemas, y relaciona su posición y distribución en el espacio con los procesos a escalas superiores (nacional y global). Es esta interrelación entre sus elementos lo que hace de la comarca geográfica un sistema válido de estudio y un espacio importante de interacción entre los individuos, las administraciones locales y el Estado.

\section{Domesticación del paisaje}

Desde el descubrimiento de la agricultura, el hombre ha transformado paisajes y ecosistemas domesticándolos con el fin de producir y aumentar las reservas de alimentos, reducir el riesgo de catástrofes naturales y promover el comercio. Al igual que en la selección de caracteres útiles de plantas y animales, la domesticación del paisaje consiste en la selección de ciertos atributos ecosistémicos deseables, por ejemplo, la producción de alimentos en un paisaje agrícola, y la consecuente alteración de otros menos deseables (Kareiva et al., 2007). La domesticación de paisajes incluye, además de la domesticación de plantas y animales, todas las 
prácticas y actividades, intencionadas o no, para transformar los ecosistemas en paisajes productivos y culturales para beneficio nuestro y de otras especies (Erickson, 2006). Así, las masas forestales gestionadas, la infraestructura para el riego, los suelos antrópicos y las vías de comunicación (carreteras, vías pecuarias, etc.) son parte de la naturaleza domesticada.

La historia está repleta de ejemplos de transformaciones de paisajes beneficiosas para el hombre y otras especies (la dehesa y el montado ibérico como casos cercanos paradigmáticos), así como de degradación y eventual abandono. En los primeros, el concepto de landesque capital (Hakansson and Widgren, 2014), es decir, "las inversiones en la tierra con una vida esperada mayor que la del ciclo de cultivo", indican que la inversión de trabajo en la tierra mejora su capacidad productiva más allá del futuro inmediato. Esta inversión de trabajo con vistas a largo plazo implica que, en esos procesos de domesticación, el paisaje y la vegetación pasaban a formar parte del sistema social (Erickson, 2006). En los segundos, la prevalencia de fenómenos de explotación, como la deforestación o el sobrepastoreo, alimentaban una espiral de degradación y la consecuente pérdida de atributos beneficiosos del paisaje.

En la actualidad asistimos a dos procesos opuestos que conducen a la pérdida de la sostenibilidad y resiliencia de muchos paisajes españoles. Por una parte, el abandono de los pueblos y de las actividades agropecuarias tradicionales en los paisajes agroforestales de montaña, que resultan en el aumento del riesgo de grandes incendios forestales (Ursino and Romano, 2014; Ferreira et al., 2016). Por otro lado, la intensificación insostenible de la agricultura para satisfacer la creciente demanda de alimentos, que está exacerbando los problemas ambientales como el consumo excesivo y la contaminación del agua, la pérdida de biodiversidad y la erosión y degradación de suelos (Tilman, 1999). Es en estos paisajes predominantemente agrícolas en donde la agroforestación, con apoyo de la investigación, puede contribuir a una domesticación más eficaz, al mantener un mejor equilibrio entre los servicios ecosistémicos de aprovisionamiento y los de apoyo, regulación y los culturales (Perfecto y Vandermeer, 2012; Galler et al., 2013).

\section{Agroforestería: servicios ecosistémicos a múltiples escalas}

La agroforestería es una disciplina que incluye prácticas y sistemas de uso del suelo que combinan deliberadamente árboles o arbustos con cultivos y/o animales domésticos, secuencial o simultáneamente (Nair, 1989). A escala de paisaje, la agroforestería se define como "un sistema dinámico de gestión de los recursos naturales que, mediante la integración de árboles en terrenos y en paisajes, diversifica y mantiene la producción con más beneficios ambientales, económicos y sociales a todos los niveles y para todos los usuarios de la tierra" (Leakey, 1996). Los sistemas agroforestales son, pues, multifuncionales, ya que proporcionan beneficios económicos, ambientales, sociales y culturales (Leakey, 1996).

La agroforestería es muy antigua, y ubicua, tanto en el Trópico como en nuestras latitudes (Brookfield and Padoch, 1994 citado por Sanchez, 1995). Las prác- 
ticas agrícolas en bosques y masas forestales, como el pastoreo o el cultivo, y la integración de árboles en los sistemas agrícolas han sido desde siempre herramientas y métodos de gestión de los recursos naturales por la población rural (Herzog, 2000; San Miguel et al., 2002; Leakey, 2012). Se estima que, actualmente, un $43 \%$ de la superficie agrícola mundial tiene al menos un 10\% de fracción de cabida cubierta (Zomer et al., 2014). Estudios recientes en Europa calculan que existen 15,4 millones de hectáreas agroforestales, lo que supone un 3,6\% de la superficie total.

En las últimas décadas, la agroforestería se ha establecido como una ciencia especializada y multidisciplinar (Sanchez, 1995). Numerosos estudios en distintas partes del mundo confirman que los sistemas agroforestales, además de ser productivos, proporcionan en mayor medida que los sistemas agrícolas cuatro importantes servicios ecosistémicos (Shibu, 2009):

1. Secuestro de carbono.

2. Conservación de la biodiversidad.

3. Fertilidad del suelo.

4. Calidad del aire y del agua.

La agroforestería es por tanto una forma de agricultura sostenible, así como de gestión forestal sostenible (Louise et al., 1999; Beddington et al., 2012; Dosskey et al., 2012).

Todo esto debería ser un aliciente para promover la agroforestería como una parte integral de los paisajes multifuncionales en todo el mundo. Sin embargo, la actual clasificación de usos del suelo, así como nuestra fuerte tendencia a la dicotomización (Gould, 2003), no dejan espacio para formas intermedias entre lo "agrícola" y lo "forestal", ni entre la agricultura y la selvicultura, lo que hace que la práctica de la agroforestería haya sido normalmente ignorada e incluso impedida por una normativa y políticas demasiado sectoriales (Van Noordwijk et al., 2008). Por ejemplo, las penalizaciones en la elegibilidad y en el cálculo del coeficiente de admisibilidad de los pastos arbolados que sufren los ganaderos para recibir ayudas de la PAC, o la falta de reconocimiento del valor forrajero de muchos árboles y arbustos para calcular la admisibilidad para los pagos del primer pilar de la PAC (Ruiz y Beaufoy, 2017).

La necesidad de la sociedad de productos agrícolas y forestales, además de servicios ambientales, pueden ser satisfechas con distintas combinaciones de bosques, plantaciones forestales intensivas y extensivas, sistemas agrícolas intensivos, así como con "mosaicos agroforestales" multifuncionales. A día de hoy, los organismos públicos sólo contemplan las opciones agrícolas o las forestales, mientras que la agroforestal sigue siendo inexistente por ignorada. Para promover la agroforestación como una alternativa a la forestación se necesitaría, en primer lugar, reconocer formalmente el uso de suelo agroforestal, así como los bienes y servicios que genera. En segundo lugar, habría que apoyar la generación de conocimiento sobre la agroforestería y su diseminación mediante procesos participativos de investigación y extensión. Y por último, sería necesario un marco normativo y políticas más 
favorables que, en lugar de penalizar la agroforestería, consiguiera ponerla al mismo nivel que los sistemas agrícolas y forestales.

\section{Participación pública}

La participación pública en la gestión forestal es el proceso por el cual los individuos, las comunidades y los grupos de interés pueden intercambiar información, expresar sus intereses e influir en las decisiones o los resultados de la planificación y la gestión forestal. En un buen proceso de participación el intercambio de información, de ideas y de conocimiento entre expertos, técnicos gestores y los grupos de interés debe ser recíproco (Beckley et al., 2005).

Actualmente existe un gran consenso en que la participación pública es esencial para la gestión forestal sostenible. Se afirma (Beckley et al., 2005; Reed et al., 2008; Metcalf et al., 2015) que resulta en una mejor toma de decisiones, pues la participación:

a) Considera fuentes de información y conocimiento local y estrategias de gestión alternativas.

b) Ayuda a crear confianza entre todas las partes implicadas y promueve el aprendizaje social.

c) Confiere legitimidad a los resultados.

d) Ayuda a percibir las decisiones ambientales como más justas, al considerar las diversas necesidades y valores de los agentes implicados.

e) Permite intervenciones y el uso de técnicas mejor adaptadas a las condiciones ambientales y socio-culturales locales.

f) Da más robustez a los resultados de la investigación.

g) Facilita la inclusión de distintas ideas y perspectivas, que de otra forma pasarían por alto, y con ello aumenta la probabilidad de que se satisfagan las prioridades y las necesidades locales.

h) Otorga a los participantes un mayor control del proceso de planificación, lo cual resulta en mayor apoyo a la implementación de decisiones.

España reconoce la necesidad de promover la participación pública en la planificación y gestión paisajística y forestal. La Convención Europea sobre el Paisaje de 2001, ratificada por nuestro país, indica en su Artículo 6 que “en los procesos de planificación del paisaje es necesario asegurar la cooperación institucional y una amplia participación pública y de las asociaciones que protegen el interés general". La Ley de Protección de la Naturaleza de 1989 y la Estrategia Forestal Española de 1999 recogen provisiones sobre la participación en relación al paisaje y a la gestión de los recursos forestales. La participación pública y social en la formulación de las políticas, estrategias y programas es uno de los principios inspiradores del Plan Forestal Español (con vigencia hasta el año 2032). A escala regional, los Planes de Ordenación de los Recursos Forestales (PORF), que son los instrumentos desarrollados por los gobiernos autonómicos para la gestión y protección 
de paisajes, deben cumplir con el principio de información y consulta pública de las diversas asociaciones y grupos de interés (Gobex, 2014).

La calidad de las decisiones tomadas mediante participación pública depende, en gran medida, de la calidad del proceso de participación (Reed et al., 2008). En España, a pesar del apoyo de la legislación, los procesos de participación pública en la gestión forestal son, en realidad, escasos y deficientes, salvo notables excepciones (Palomo et al., 2011; Palacios-Agúndez et al., 2013; Bruña-García y Marey-Pérez, 2014). Por una parte, la participación no está institucionalizada en nuestros órganos de administración, con lo que el poder de decisión para la planificación y gestión forestal sigue estando, como en el pasado, exclusivamente en manos de técnicos y expertos del Estado. Por otra parte, la información y consulta, formas de participación pública contempladas en los PORF, no son instrumentos de participación adecuados para conferir a los interesados poder de decisión o de influencia en decisiones ya tomadas (Arnstein, 1969). Frecuentemente, las consultas acaban siendo sólo una mera formalidad para informar a la población sobre decisiones previamente tomadas, cuando en realidad los procesos participativos, para ser efectivos, deben ser iterativos y facilitados por extensionistas expertos capaces de incorporar un amplio conjunto de valores a los debates y a la toma de decisiones (Beckley et al., 2005). La toma de decisiones centralizada sin un proceso genuino de participación con el que considerar el conocimiento, las necesidades y los valores de la población local acaba generando, a largo plazo, hostilidad y desapego por un recurso forestal que es considerado como ajeno (Metcalf et al., 2015).

Los paisajes agrícolas están directamente influenciados por las decisiones de una multitud de agentes y grupos de interés (agricultores, ganaderos, organizaciones diversas, instituciones públicas locales). La mejora y diversificación de estos paisajes requiere, por tanto, considerar el comportamiento de todas las partes interesadas. Sin embargo, esto no se puede conseguir sin negociación (más que planificación), acuerdos y consensos (Sayer et al., 2008; Derak et al., 2018). La inversión de tiempo y esfuerzo en poner en marcha una estrategia participativa adecuada y amplia para desarrollar y negociar escenarios compartidos del paisaje futuro, así como para evaluar los resultados en el paisaje de las acciones acordadas es fundamental si queremos tener paisajes más sostenibles.

\section{Conclusión}

La diversificación y mejora de los paisajes agrícolas mediante la forestación necesita de una estrategia con una visión holística e integradora de los problemas y la realidad de los agroecosistemas, en lugar de la prevalente visión dicotómica y sectorial que separa lo agrícola de lo forestal. Para que sea efectiva en la provisión de los bienes y los servicios ecosistémicos que la sociedad demanda, esta estrategia debe implementarse a escala comarcal y facilitar la participación de los distintos agentes y grupos de interés en la planificación de un proceso de domes- 
ticación del paisaje rural basado en la agroforestería. Para ello se necesitan técnicos y agentes de extensión y desarrollo rural con capacidad para llevar a cabo un trabajo multidisciplinar y de involucrar a la población rural en la toma de decisiones y en las acciones necesarias para conseguir paisajes agrícolas más sostenibles.

\section{Bibliografía}

Arnstein, S.R.; 1969. A Ladder Of Citizen Participation, Journal of the American Planning Association. 35 (4), 216 - 224. https://doi.org/10.1080/01944366 908977225

Balint, P.J.; Stewart, R.E.; Desai, A.; Walters, L.C.; 2011. Wicked Environmental Problems. Managing uncertainty and conflict. Island Press, Washington D.C. https://doi.org/10.58 22/978-1-61091-047-7

Barral, M.P.; Benayas, J.M.; Meli, P.; Maceira, N.O.; 2015. Quantifying the impacts of ecological restoration on biodiversity and ecosystem services in agroecosystems: A global meta-analysis. Agric. Ecosyst. Environ. 202, 223-231. https://doi.org/10.1016/j. agee.2015.01.009

Beddington, J.; Asaduzzaman, M.; Clark, M.; Fernandez, A.; Guillou, M.; Jahn, M.; Erda, L.; Mamo, T.; Van Bo, N.; Nobre, C.A.; Scholes, R.; Sharma, R.; Wakhungu, J.; 2012. Achieving food security in the face of climate change: Final report from the Commission on Sustainable Agriculture and Climate Change. CGIAR Research Program on Climate Change, Agriculture and Food Security (CCAFS). Copenhagen, Denmark. http://www.ccafs.cgiar.org/commission Accessed 8 September 2018.

Beckley, T.; Parkins, J.; Sheppard, S.; 2005. Public Participation in Sustainable Forest Management: A Reference Guide. Sustainable Forest Management Network, Edmonton, Alberta. https://www.researchgate.net/publication/238726836_Public_Participation_ in_Sustainable_Forest_Management_A_Reference_Guide Accessed 3 November 2018.

Bruña-García, X.; Marey-Pérez, M.F;. 2014. Public participation: a need of forest planning. iForest 7: 216-226. http://www.sisef.it/iforest/contents/?id=ifor0979-007 https://doi. org/10.3832/ifor0979-007

DeFries, R.; Nagendra, H.; 2017. Ecosystem management as a wicked problem. Science 356, 265-270. https://doi.org/10.1126/science.aal1950

den Herder, M.; et al., 2017. Current extent and stratification of agroforestry in the European Union. Agric. Ecosyst. Environ. 241, 121-132. https://doi.org/10.1016/j.agee.20 17.03.005

Derak, M.; Cortina, J.; Taiqui, L.; Aledo, A.; 2018. A proposed framework for participatory forest restoration in semiarid areas of North Africa. Restoration Ecol. 26 (1), 18-25. https://doi.org/10.1111/rec.12486

Dosskey, M.G.; Bentrup, G.; Schoeneberger, M.; 2012. A Role for Agroforestry in Forest Restoration in the Lower Mississipi Alluvial Valley. J. For. 110 (1): 48-55. https://doi. org/10.5849/jof.10-061

Erickson, C.L.; 2006. The domesticated landscapes of the Bolivian Amazon. In: Balee, W.; Erickson, C.L.; (eds.), Time and Complexity in Historical Ecology: Studies in the Neotropical Lowlands. Columbia University Press, New York, pp. 235-278. https://doi. org/10.7312/bale13562-011 
Estrategia Forestal Española. En: (https://www.mapa.gob.es/es/desarrollo-rural/temas/ poli tica-forestal/planificacion-forestal/politica-forestal-en-espana/pfe_estrategia_ forestal. aspx). Accessed 5 November 2018.

FAO, JRC, 2012. Global forest land-use change 1990-2005. FAO Forestry Paper No. 169. Food and Agriculture Organization of the United Nations and European Commission Joint Research Centre. Rome, FAO. http://www.fao.org/docrep/017/i3110e/i3110e.pdf Accessed 2 October 2018

Ferreira-Leite, F.; Bento-Gonçalves, A.; Vieira, A.; Nunes, A.; Lourenço, L.; 2016. Incidence and recurrence of large forest fires in mainland Portugal. Nat Hazards 84:1035-10 53. https://doi.org/10.1007/s11069-016-2474-y

FOREST EUROPE, 2015. State of Europe's Forests 2015. https://www.foresteurope.org/ docs/fullsoef2015.pdf Accessed. 25 August 2018.

Galler, C.; von Haaren, C.; Albert, C.; 2013. Chapter 13 Planning Multifunctional Measures for Efficient Landscape Management: Quantifying and Comparing the Added Value of Integrated and Segregated Management Concepts. In: Fu, B.; and Jones, K.B.; (eds.), Landscape Ecology for Sustainable Environment and Culture: 249-284. https://doi.org/ 10.1007/978-94-007-6530-6_13

García del Barrio, J.M.; Bolaños, F.; Elena-Roselló, R.; 2003. Clasificación de los paisajes rurales españoles según su composición espacial. Invest. Agrar. Sist. Recur. For. 12(3): 5-17.

Gould, S.J.; 2003. The Hedgehog, the Fox and the Magister's Pox. Mending the gap between science and the humanities. Three River Press, New York. https://doi.org/10.4159/har vard.9780674063402

Gobex 2014. Manual del Sistema de Gestión Forestal Sostenible de los montes de Gestión Pública de la zona noroccidental de Extremadura. http://extremambiente.juntaex.es/ files/forestal/Decreto\%20247_2013/DOC_GFS_1_MANUAL\%20DEL\%20SISTEMA \%20Gesti\%C3\%B3n\%20Forestal\%20sostenible.pdf. Accessed 2 October 2018.

Hakansson, N.T.; Mats Widgren, M.; (eds.), 2014. Landesque capital: the historical ecology of enduring landscape modifications. Left Coast Press.

Herzog, F.; 2000. The importance of perennial trees for the balance of northern European agricultural landscapes. Unasylva. 200 (51), 42-48.

Ilstedt, U.; Bargués Tobella, A.; Bazié, H.R.; Bayala, J.; Verbeeten, E.; Nyberg, G.; Sanou, J.; Benegas, L.; Murdiyarso, D.; Laudon, H.; Sheil, D.; Malmer, A.; 2016. Intermediate tree cover can maximize groundwater recharge in the seasonally dry tropics. Sci. Rep. 6, 21930. https://doi.org/10.1038/srep21930

INE 2016. https://www.ine.es/prodyser/pubweb/anuario17/anu17_12agric.pdf Accessed 9 November 2018.

Kareiva, P.; Watts, S.; McDonald, R.; Boucher, T.; 2007. Domesticated nature: shaping landscapes and ecosystems for human welfare. Science, 316, 1866-1869. https://doi. org/10.1126/science.1140170

Laestadius, L.; Maginnis, S.; Minnemeyer, S.; Potapov, P.; Saint-Laurent, C.; Sizer, N.; 2012. Mapping opportunities for forest landscape restoration. Unasylva (FAO) 62: 238. http://www.wri.org/resources/maps/atlas-forest-and-landscape-restoration-opportuni ties. Accessed. 10 Octubre 2018. https://doi.org/10.4324/9781849773010

Leakey, R.R.B.; 1996. Definition of agroforestry revisited. Agroforestry Today, 8(1), 5-7.

Leakey, R.R.B.; 2012. Living with the trees of life: towards the transformation of tropical agriculture. CABI, Oxfordshire. https://doi.org/10.1079/9781780640990.0000 
Louise, E.; Buck, J.P.; Lassoie, E.; Fernandes, C.M.; 1999. Agroforestry in sustainable agricultural systems. CRC PRESS, Boca Raton, Florida.

Manning, A.D.; Fischer, J.; Lindenmayer, D.B.; 2006. Scattered trees are keystone structures - Implications for conservation. Biol Conserv 132: 311-321. https://doi.org/10.1016/j. biocon.2006.04.023

MAPA, 2006. Forestación de Tierras Agrícolas: análisis de su evolución y contribución a la fijación del carbono y al uso racional de la tierra. Ministerio de Agricultura, Pesca y Alimentación. Madrid.

Mata, R.; Panadero, M.; Pillet, F., Sancho, J.; Tapiador, F.J.; del Pozo, C.; 2004. Atlas de los Paisajes de Castilla-La Mancha. Universidad de Castilla-La Mancha. https://www.cas tillalamancha.es/sites/default/files/documentos/pdf/20170330/atlas-clm.pdf Accessed 3 September 2018

Mather, A.S.; 2004. Forest transition theory and the reforesting of Scotland. Scott. Geogr. J. 120: 83-98. https://doi.org/10.1080/00369220418737194

Mather, A.S.; 2007. Recent Asian forest transition in relation to forest transition theory. Int. For. Rev. 9: 491-502. https://doi.org/10.1505/ifor.9.1.491

Mather, A.S.; Needle, C.L.; 1998. The forest transition: A theoretical basis. Area 30: 117124. https://doi.org/10.1111/j.1475-4762.1998.tb00055.x

McNeely, J.A.; 1998. Economics and Biological Diversity: Developing and Using Economic Incentives to Conserve Biological Resources. IUCN. Gland, Switzerland.

Metcalf, E.C.; Mohr, J.J.; Yung, L.; Metcalf, P.; Craig, D.; 2015. The role of trust in restoration success: public engagement and temporal and spatial scale in a complex socialecological system. Restoration Ecol. 23:315-324. https://doi.org/10.1111/rec.12188

Montiel, C.; Galiana, L.; 2004. La Restauración de Paisajes Forestales a través de la Forestación de Tierras Agrarias. Actas de la III Reunión sobre Repoblaciones Forestales. Cuad. Soc. Esp. Cien. For. 17: 193-198.

Nair, P.K.R.; 1989. Agroforestry defined. In: Nair P.K.R. (ed.) Agroforestry Systems in the tropics. Kluwer Academic Publishers, Dordrecht, Netherlands, 13-18. https://doi.org/ 10.1007/978-94-009-2565-6_2

Palacios-Agundez, I.; Casado-Arzuaga I.; Madariaga I.; Onaindia M.; 2013. The relevance of local participatory scenario planning for ecosystem management policies in the Basque Country, northern Spain. Ecology and Society 18(3): 7. https://doi.org/10.5751/ ES-05619-180307

Palomo, I.; Martín-López B.; López-Santiago C.; Montes C.; 2011. Participatory scenario planning for protected areas management under the ecosystem services framework: the doñana social-ecological system in southwestern Spain. Ecology and Society 16(1): 23. http://www.ecologyandsociety.org/vol16/iss1/art23/ https://doi.org/10.5751/ES-03862 $-160123$

Pemán, J.; Vadell, E.; 2009. Reconstrucción de la estadística de la actividad repobladora desde 1879 hasta nuestros días. $5^{\circ}$ Congreso Forestal Español.

Pereira, M.; Rodríguez, A.; 2010. Conservation value of linear woody remnants for two forest carnivores in a Mediterranean agricultural landscape. J Appl Ecol 47, 611-620. https://doi.org/10.1111/j.1365-2664.2010.01804.x

Perfecto, I.; Vandermeer, J.; 2012. Separación o integración para la conservación de biodiversidad: la ideología detrás del debate "land-sharing" frente a "land-sparing". Ecosistemas 21(1-2), 180-191. 
Reed, M.S.; 2008. Stakeholder participation for environmental management: A literature review. Biol Cons. 141, 2417-2431. https://doi.org/10.1016/j.biocon.2008.07.014

Reed, J.; Van Vianen, J.; Deakin, E.L.; Barlow, J.; Sunderland, T.; 2016. Integrated landscape approaches to managing social and environmental issues in the tropics: learning from the past to guide the future. Global Change Biol. 22, 2540-2554. https://doi.org/10.11 $11 / \mathrm{gcb} .13284$

Reque, J.; Pérez, R.; 2011. Del Monte al Rodal. Manual SIG de Inventario Forestal. Universidad de Valladolid - Vicerrectorado de Docencia, Valladolid, España. http://www.pfc yl.es/documento/del-monte-al-rodal-manual-sig-de-inventario-forestal

Rittel, H.W.J.; Webber, M.M.; 1973. Dilemmas in a General Theory of Planning. Policy Sciences 4, 155-169. https://doi.org/10.1007/BF01405730

Roshetko, J.M.; Lasco, R.D.; Angeles, M.D.; 2007. Smallholder agroforestry systems for carbon storage. Mitig Adapt Strat Glob Change 12(2), 219-242. https://doi.org/10.1007 /s11027-005-9010-9

Ruiz, J.; Beaufoy, G.; 2017. Informe sobre la elegibilidad para pagos directos de la PAC de los pastos leñosos españoles. Fundación Entretantos. Accedido en: http://www.ganade riaextensiva.org/InformeElegibilidadPastos.pdf Accessed 8 November 2018.

Sanchez, P.A.; 1995. Science in agroforestry. Agrofor Syst. 30:5-55. https://doi.org/10.1007 /978-94-017-0681-0_1

San Miguel, A.; S. Roig S., Cañellas, I., 2002. Las prácticas agroforestales en la Península Ibérica. Actas de la I Reunión sistemas agroforestales-I Reunión espacios naturales. Cuad. Soc. Esp. Cien. For. 14, 33-38.

Sayer, J.; Buck, L.; Dudley, N.; 2008. What is a landscape approach? Learning from landscapes, arborvitae special issue. Gland, Switzerland: IUCN. http:/www.iucn.org/about /work/programmes/forest/?1544/arborvitae-special-issue-Learning-from-Landscapes Accessed 3 November 2018.

Serrada, R.; Navarro, R.; Pemán, J.; 2005. La calidad de las repoblaciones forestales: una aproximación desde la selvicultura y la ecofisiología. Invest Agrar: Sist Recur For 14 (3), 462-481. https://doi.org/10.5424/srf/2005143-00943

Shibu, J.; 2009. Agroforestry for ecosystem services and environmental benefits: an overview. Agroforest Syst 76:1-10. https://doi.org/10.1007/s10457-009-9229-7

Tilman, D.; 1999. Global environmental impacts of agricultural expansion: The need for sustainable and efficient practices. Proc. Natl. Acad. Sci. USA. Vol. 96, 5995-6000. https://doi.org/10.1073/pnas.96.11.5995

Torralba, M.; Fagerholm, N.; Burgess, P.J.; Moreno, G.; Plieninger, T.; 2016. Do European agroforestry systems enhance biodiversity and ecosystem services? A meta-analysis. Agricul, Ecosys Environ. 230: 150-161. https://doi.org/10.1016/j.agee.2016.06.002

Ursino, N.; Romano, N.; 2014. Wild forest fire regime following land abandonment in the Mediterranean region, Geophys. Res. Lett., 41, 8359-8368. https://doi.org/10.1002/20 14GL061560

Van Noordwijk, M.; Roshetko, J.M.; Murniati3, Angeles, M.D.; Suyanto, Fay, C.; Tomich, T.P.; 2008. Farmer Tree Planting Barriers to Sustainable Forest Management. In: Snelder, D.J., Lasco, R.D., (eds.), Smallholder Tree Growing for Rural Development and Environmental Services. Lessons from Asia. Springer. https://doi.org/10.1007/978-14020-8261-0_20

Wortley, L.; Hero, J.M.; Howes, M.; 2013. Evaluating Ecological Restoration Success: A 
Review of the Literature. Restoration Ecol. 21(5), 537-543. https://doi.org/10.1111/ rec. 12028

Zomer, R.J.; Trabucco, A.; Coe, R.; Place, F.; van Noordwijk, M.; Xu, J.C.; 2014. Trees on farms: an update and reanalysis of agroforestry's global extent and socio-ecological characteristics. Working Paper 179. Bogor, Indonesia: World Agroforestry Centre (ICRAF) Southeast Asia Regional Program. https://doi.org/10.5716/WP14064.PDF 